\title{
Viewpoint
}

\section{Taking advantage of nature for a greener nonvolatile memory}

\author{
Markus Münzenberg \\ I. Physikalisches Institut, Universität Göttingen, 37077 Göttingen, Germany
}

\author{
Jagadeesh S. Moodera \\ Francis Bitter Magnet Laboratory, Massachusetts Institute of Technology, Cambridge, MA 02139, \\ $U S A$
}

Published March 1, 2010

Thermal magnetic fluctuations, usually thought of as a barrier to magnetic information storage and processing, can be harnessed to make green, low-energy magnetic applications a possibility.

Subject Areas: Magnetism, Nonlinear Dynamics, Spintronics

\author{
A Viewpoint on: \\ Single-Shot Time-Domain Studies of Spin-Torque-Driven Switching in Magnetic Tunnel Junctions \\ Y.-T. Cui, G. Finocchio, C. Wang, J. A. Katine, R. A. Buhrman, and D. C. Ralph \\ Physical Review Letters 104, 0972012010 - Published March 1, 2010
}

Writing information onto nanoscale magnetic oscillators as magnetic bits presents many challenges, some of which become more formidable as we pursue higherdensity information storage. It is true that the electric current densities needed at present to rotate the magnetization direction are lower than those needed in traditional semiconductor devices, where information has to be constantly rewritten. However, the needed currents are still large, resulting in high-energy consumption and Joule heating during writing. Additionally, reducing the magnetic device size generally results in unwanted thermal fluctuations, which have to be overcome by employing high anisotropy material to keep the bit stable.

The mechanism responsible for the transfer of magnetic information is spin-transfer torque, which arises whenever a spin current created by one magnetic thin film flows through another magnetic film whose moment is not collinear with the first. In the process of spin filtering, the second film necessarily picks up part of the spin angular momentum carried by the electron spins from the first film. How does this happen? The magnetization of the ferromagnet exerts a torque on the flowing electron spins, aligning them to the local magnetization direction. Momentum conservation results in equal and opposite torque on the second ferromagnet, the so-called free layer. This spin-transfer-torque-induced magnetization switching, independently predicted in 1996 by both John Slonczewski [1] and Luc Berger [2], and experimentally observed by Maxim Tsoi et al. [3], is now being extensively explored both for probing the fundamental physics of magnetic dynamics, and for applications in storage technology. For storage, information is stored in the magnetization direction of the free layer, in its two stable states [Fig. 1 (a)]. This is seen as an ideal way to

DOI: $10.1103 /$ Physics.3.19

URL: http://link.aps .org/doi/10.1103/Physics.3.19 a) Non-thermal

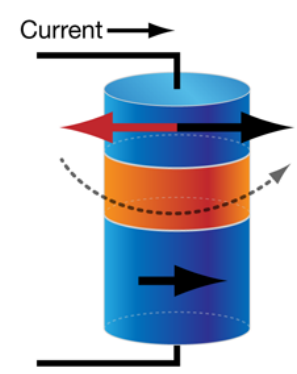

b) Thermally assisted

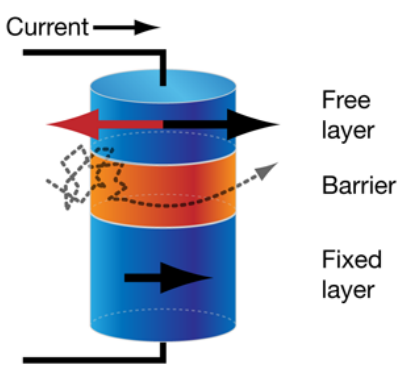

FIG. 1: Schematic trajectory of the transition from the antiparallel to parallel state in (a) regular spin-transfer torque switching and (b) thermally assisted. Black and red-black arrows represent the magnetization of the layer. (Illustration: Alan Stonebraker)

write information into magnetic random access memories (MRAMs) leading to enormous power savings, in addition to fast, nonvolatile, and ultrahigh-density memory storage.

For memory applications, one wants the individual electron spins of the magnetic element to behave as if they comprised a giant spin (called macrospin). The magnetic anisotropy energy defines the height of the energy barrier that must be overcome for writing, as well as the stability of the bit, and as such are two competing effects. Magnetic storage by spin-torque-transfer methods is an area of intense research, the challenge being to stabilize the written bit just right so that the statistical fluctuations of the impinging electron spin current can take it from one stable orientation to the other.

(C) 2010 American Physical Society 


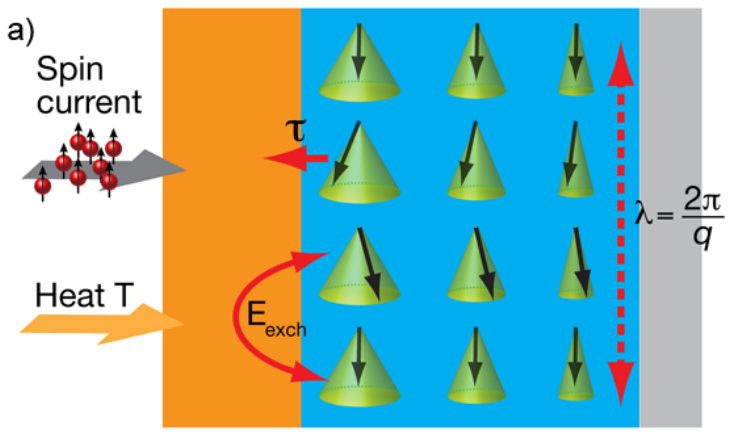

Free layer

b)
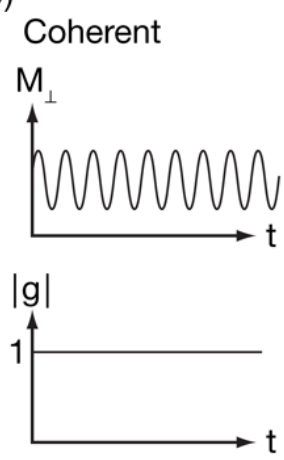

Thermal

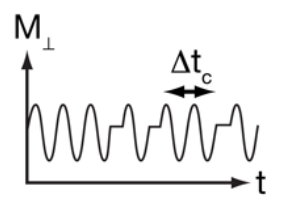

$|g|$

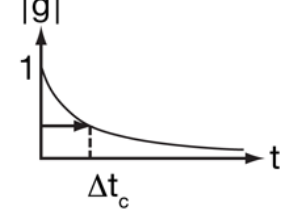

FIG. 2: (a) Spin current (gray) and heat (beige) currents, and fluctuations of the magnetization, appearing on all length scales for heating. In (b) the analysis of coherent precession excited in regular spin-transfer torque switching is depicted. In the thermal regime, dephasing is found [right side, (b)]. This results in a loss of correlation and the correlation function $|g|$, derived from the contrast of the autocorrelation, decreases on a characteristic time scale $\Delta t_{c}$ which allows a quantitative access. (Illustration: Alan Stonebraker)

How realistic is the macrospin model? On the face of it, this simplification presents a problem because these devices are intrinsically inhomogeneous [Fig. 2 (a)]. Fluctuations are inherent to spin transfer switching and the magnetic behavior of small particles, since randomly impinging electrons in the tunnel process transfer the spin torque at the interface to a depth of about $1 \mathrm{~nm}$. In addition, in the tunneling process itself, magnons (quantized spin waves) are excited at the interface [4, 5. and Joule heating increases the thermal population of magnons. However, above a certain population of magnons, the picture is better described by a fluctuating domains image [6. Although the underlying physics is inhomogeneous and complex, simplified models assuming the magnetization as homogenous and rigid have been very successful so far. The principal reason is that the exchange energy coupling the spins together flattens out the magnetic fluctuations, and thus the simple macrospin model is quite reasonable for many applications.

However, strong deviations from the macrospin pic-

ture have been found in time-resolved x-ray magnetic dichroism imaging of the magnetization in structured nanopillars. These studies showed that the magnetization is inhomogeneous and that vortexlike structures dominate the curled magnetization during the switching process [7]. Some time ago the suggestion was made to include stochastic fluctuations that mirror the adequate thermodynamic spin statistics of the ferromagnet in order to understand the complex mode spectra observed in these devices. The quantitative experimental access to nondeterministic fluctuations in experiments has been limited so far. Signal averaging techniques have been used to trace the dynamics of the excited magnetic elements because the signals are low. No method so far has allowed subnanosecond resolution in a single-shot experiment, though pioneering experiments by Devolder et al. [8] have already shown the nondeterministic nature of the switching events.

In a paper in Physical Review Letters 9], Yongtao Cui, Chen Wang, Robert Burhman, and Daniel Ralph at Cornell University, US, Giovanni Finocchio at the University of Messina, Italy, and Jordan Katine at Hitachi Laboratories, US, report on a technique that substantially improves the sensitivity of single-shot resistance measurements, allowing for the first time the direct observation of the magnetic fluctuations both prior to and during spintorque switching. This detailed view of the magnetic dynamics allows improvement of the switching process and also makes magnetic tunnel junctions promising models for controlling nonlinear systems excited by thermal fluctuations. Taking advantage of magnetic fluctuations and switching the magnetic bit by a small spin-polarized current may be an elegant way towards low-energy consuming, "green" information technologies.

Cui et al. vividly show the highest resolution mapping of magnetization fluctuations resulting in a final switching event. They used magnetic junctions with high magnetoresistance (TMR) signal and a clever differential measurement scheme (which involves splitting their pulse and subtracting the inverted signal pulse afterwards), allowing them to make single-shot measurements with high precision. They studied the onset of magnetization fluctuations in the free layer after the voltage pulse was applied in two initial antiparallel geometries: one with perfect antiparallel initial configuration and a second one with the free layer rotated by $\sim 15^{\circ}$ from the antiparallel initial configuration using a small field applied along the hard-axis. For voltages applied across the junction, the spin-transfer torque alone should not have been sufficient to rotate the magnetization from antiparallel to the parallel state, but nevertheless, the authors observed nondeterministic switching. The switching events showed a broad time distribution ranging from one to one hundred nanoseconds. This kind of time distribution is the signature of a thermally assisted switching, meaning that thermal fluctuations of the magnetization are needed [Fig. 1 (b)]. Interestingly, the averaged signal, which is measured conventionally, shows an onset behavior of the os- 
cillation. In the single trajectory traces, however, even the detailed onset of fluctuations finally leading to the switching looks very different for each switching event, and it is unpredictable at what time the nanometer sized magnetic element will switch. Here, standard macrospin concepts collapse.

To support the differing observations of Cui et al., micromagnetic simulations were performed for both geometries. They show beautifully that at a high magnon density, magnons couple to yield nanometer-scale fluctuations. This means that magnons in the $1-20 \mathrm{THz}$ frequency range, corresponding to nanometer wavelengths, are highly interacting, and couple to the eigenmode spectra determined by the shape of the element. In the perfect antiparallel configuration, an inhomogeneous growth of a nucleation over a critical size spreads over the element, switching the magnetization. The switching scenario differs, however, in the second case. If the field is applied and the free layer rotated by $\sim 15^{\circ}$ from the antiparallel initial configuration, the switching is more homogeneous and the macrospin picture is more valid.

The high-precision experiments allowed Cui et al. to access this stochastic, heat-driven process systematically. The autocorrelation trace of the preswitching conductance variations reveal the coherence time $\Delta t_{c}$ of the thermal and current driven fluctuations, similar to coherence of waves in optics. The coherence decay quantifies the degree of temporal coherence, shown in Fig. 2 (b). The magnetic fluctuations found by Cui et al. are stochastic and decay with a characteristic time scale $\Delta t_{c}$. The temperature and spin-transfer torque, which works as an inverse damping, promote the fluctuations [Fig. 2 (a)], which build up in a switching event, overcoming the barrier to the parallel configuration.

What advantage can we gain by making use of this heating? Surprisingly, the use of thermal fluctuations physics to overcome a switching barrier, applying a small asymmetry to move the system over the barrier, is not unique, and can be found in complementary experiments using intense femtosecond laser pulses [10. Just recently it was reported that the circular polarization of the femtosecond pulse takes the role of the spin-polarized current, by giving the system a kick. Passing through a state of quenched magnetization seems to be the key to obtaining ultimate magnetic switching speed [11. As shown theoretically, by incorporating temperature effects that reduce the absolute value of the averaged magnetization, this may be a way to keep descriptions in a macrospin picture simple and enabling technically relevant implementation of thermally assisted writing concepts in the future [11, 12]. This allows one to take advantage of nature, in this case the intrinsic magnetic fluctuations in the magnetic nanometer-sized elements, to reduce writing currents in future MRAM devices.

\section{References}

[1] J. C. Slonczewski, J. Magn. Magn. Mater. 159, L1 (1996).

[2] L. Berger, Phys. Rev. B 54, 9353 (1996).

[3] M. Tsoi et al., Phys. Rev. Lett. 80, 4281 (1998).

[4] J. S. Moodera et al., Phys. Rev. Lett. 80, 2941 (1998).

[5] V. Drewello et al., Phys. Rev. B 77, 014440 (2008).

[6] S. V. Halivov et al., Phys. Rev. B. 58, 293 (1998).

[7] Y. Acremann et al., Phys. Rev. Lett. 96, 217202 (2006).

[8] T. Devolder et al., Phys. Rev. Lett 100, 057206 (2008).

[9] Y-T. Cui, G. Finocchio, C. Wang, J. A. Katine, R. A. Buhrman, and D. C. Ralph, Phys. Rev. Lett. 104, 097201 (2010).

[10] M. Dordjevic et al., Phys. Rev. B 75, 012404 (2007).

[11] K. Vahaplar et al., Phys. Rev. Lett. 103, 117201 (2009); R. Hertel, Physics 2, 73 (2009).

[12] D. A. Garanin, Phys. Rev. B 55, 3050 (1997); P. M. Haney, and M. D. Stiles, Phys. Rev. B 80, 094418 (2009). 


\section{About the Authors}

\section{Markus Münzenberg}

Markus Münenberg is leading a research group at Göttingen University and is a professor
of experimental physics. He received his Ph.D. from Göttingen University in 2000 on x-ray
magnetic circular dichroism experiments at European synchrotron facilities. He performed
his post-doctoral research with Jagadeesh S. Moodera's group at the Massachusetts Institute
of Technology (MIT) Cambridge, US, from 2001 to 2002 , on spin transport in ferromagnetic
tunneling magnetoresistance (TMR) structures, and started a femtosecond magnetization
dynamics group as a Junior faculty from 2002 to 2008 at Göttingen University, where he is
currently appointed. His interests combine spin-transport and dynamics.

\section{Jagadeesh S. Moodera}

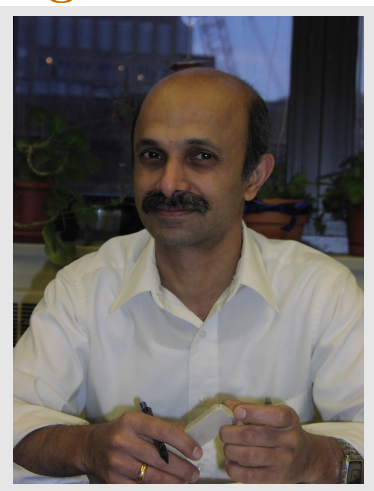

Dr. Jagadeesh S. Moodera is a scientist and leader of the Thin Film Magnetism, Tunneling and Nano Spintronics group at the Francis Bitter Magnet Laboratory, Massachusetts Institute of Technology. He is also a visiting professor at the Applied Physics Department, Eindhoven Technical University (the Netherlands). He obtained his Ph.D. from the Indian Institute of Technology, Madras. His current research interests include fundamental phenomena in magnetism, particularly spin-polarized tunneling, superconductivity, and nanospintronics. 